\title{
The sound absorption property of microperforated panel with partitioned cavity
}

\author{
GAI Xiao-ling, LI Xian-hui, ZHANG Bin, Liu Yan-Qi, XING Tuo \\ Beijing Key Laboratory of Environment Noise and Vibration, Beijing Municipal Institute of Labor \\ Protection, Beijing 100054, China \\ email: gxldynmg@163.com, lixianh@dlut.edu.cn, zhangbin815@hotmail.com, jdygliu@163.com, \\ xingtuo1991@163.com
}

Keywords: microperforated panel, partitioned cavity, sound absorption coefficient, sound absorption peak, sound absorption bandwidth

\begin{abstract}
In order to broaden the sound absorption bandwidth of single-leaf microperforated panel(MPP),The structure of MPP with partitioned cavity is introduced and its sound absorption performance is studied by impedance tube experiment. Results show that the MPP with partitioned cavity has two sound absorption peaks. Meanwhile, the sound absorption bandwidth is broaden and the sound absorption performance of the low and middle frequency is improved.
\end{abstract}

\section{Introduction}

With the development of economic construction, urban noise is more and more serious; the noise becomes an issue of popular concern. Reduce noise and create a beautiful acoustic environment is an important element to improve the quality of life of the people. A microperforated panel (MPP) absorber has become widely known as the most attractive alternative for the next generation sound absorbing material [1]. The MPP is first proposed by Maa, who has established its theoretical basis and design principle [2-4]. The MPP absorber is a thin panel or membrane less than $1 \mathrm{~mm}$ thick with perforation of less than $1 \%$ perforation ratio with air-back cavity and a rigid backing [5]. The fundamental absorbing mechanism of the MPP absorber, which is typically backed by an air cavity and a rigid wall, is Helmholtz-resonance absorption [6]. This type of absorption is mainly due to frictional loss in the air flow of the apertures [6]. With the rapid development of processing technologies and computational methods, micro-perforated panel sound absorption theory has also been further development [7-9]. But usually the single-leaf MPP sound absorbing structure is generally only one resonance absorption peak. Although, the absorption coefficient of absorption peak is able to control above 0.9, but the sound absorption bandwidth is usually limited to about two octaves [2-4]. In order to broaden the absorption frequency range, Maa has proposed a double-leaf MPP backed by a rigid-back wall with an air-cavity [10]. Recently, Asdrubali and Pispola have studied this type of absorber for its application to noise barriers [11]. This absorber is intended to produce two resonators so that a broader absorption frequency range can be obtained. The acoustical properties of a structure composed of two parallel MPP with an air-cavity between them and no rigid backing is studied numerically by Sakagami [12].

Although lots of researches have been done on broaden the absorption frequency range of MPP, it appears that no relevant reports have been given for the MPP with partitioned cavity. The main purpose of this paper will focus on the acoustic performance studies of MPP absorbers with partitioned cavity. Structure of this paper will be arranged as follows: In Section 2, sample of the MPP with partitioned cavity will be shown. In Section 3, the sound absorption performance of MPP absorber without a rigid backing will be studied. Finally, the conclusions will be given in section 4 .

\section{Sample construction}

MPP absorbers with partitioned cavity are constructed by a MPP and a structure of partitioned cavity. MPP used is aluminum panel $100 \mathrm{~mm}$ in diameter. Thick, porosity and aperture for the MPP are $0.8 \mathrm{~mm}, 1 \%$ and $0.8 \mathrm{~mm}$, respectively. Figure 1 shows the structure of MPP. The structure of 
partitioned cavity is added to MPP by attaching. Figure 2 shows the structure of MPP with partitioned cavity. The deep of partitioned cavity is $30 \mathrm{~mm}$.

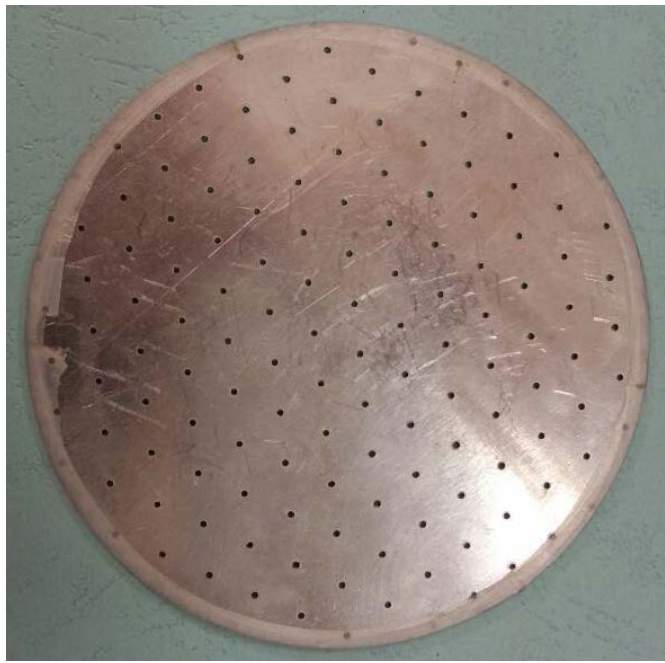

Figure 1. microperforated panel

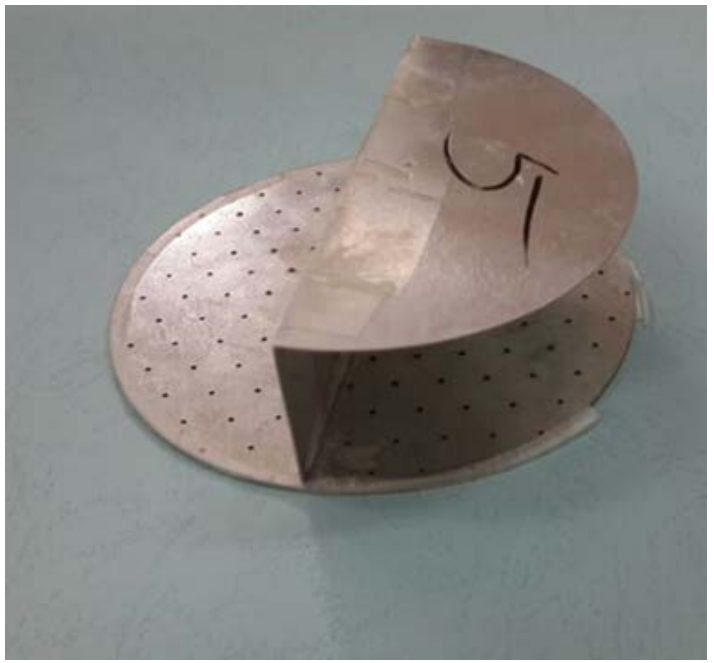

Figure 2. microperforated panel with partitioned cavity

\section{Impedance tube experiments}

We study the property of MPP with partitioned cavity by impedance tube experiments. Figure 3 is the test system of impedance tube. Figure 4 compare the normal incidence sound absorption coefficient of the single-leaf MPP and MPP with partitioned cavity. The deeps of backing cavity are $50 \mathrm{~mm}$ in impedance tube experiments. Through figure 4, we find that the single-leaf MPP has one sound absorption peaks at $756 \mathrm{~Hz}$. However, the MPP with partitioned cavity has two main sound absorption peaks at $536 \mathrm{~Hz}$ and $1336 \mathrm{~Hz}$ respectively. In addition, there also exists another peak 0.579 at $936 \mathrm{~Hz}$ on the structure of the MPP with partitioned cavity. The reason may be the vibration of partitioned plate. Meanwhile, the maximum sound absorption peak of MPP with partitioned cavity is 0.988 is higher than 0.960 of the single-leaf MPP's. The sound absorption coefficient of the MPP with partitioned cavity over 0.4 in the frequency range $256-1600 \mathrm{~Hz}$, which is much wider than 396-1416 Hz of the single-leaf MPP. If the partitioned cavities are well designed, it will improve the sound absorption performance of the partitioned cavity.

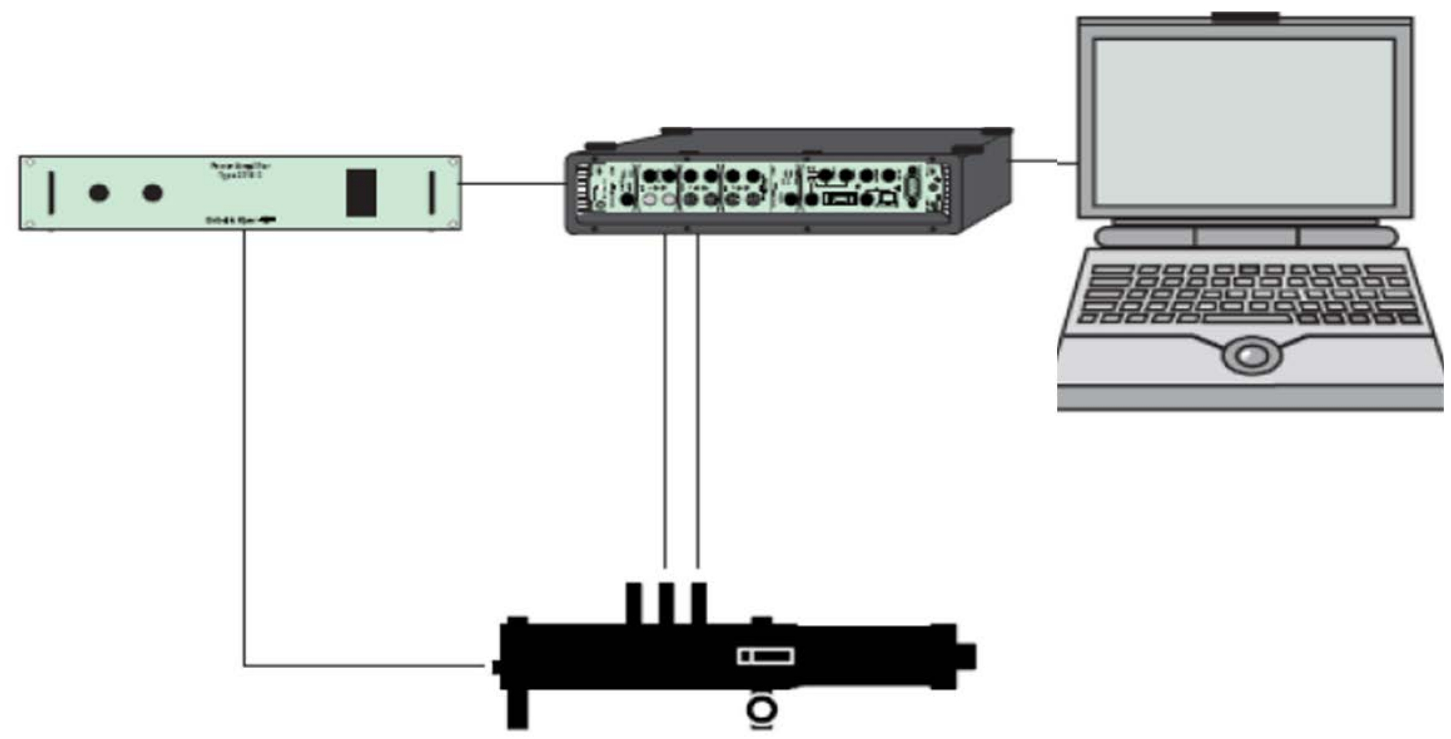

Figure 3. The test system of impedance tube 


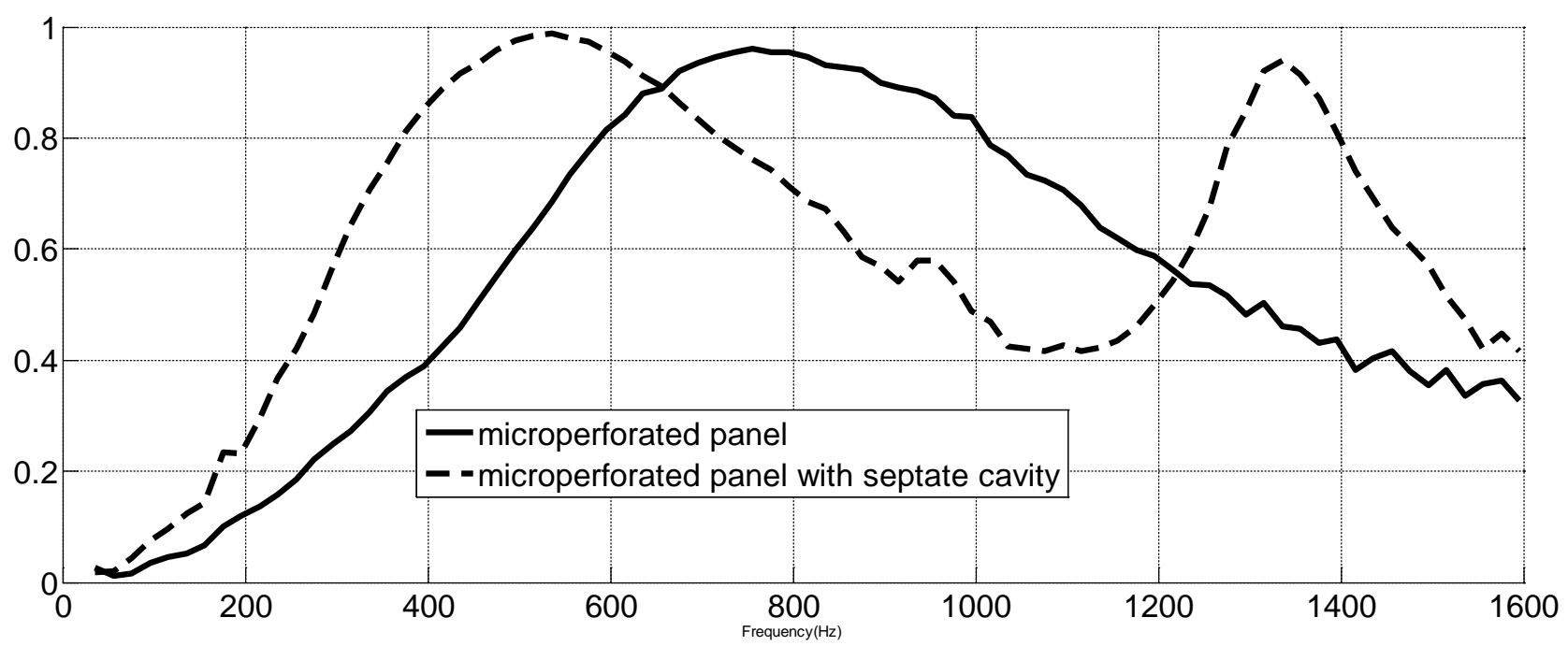

Figure 4. the normal incidence sound absorption coefficient of microperforated panel and microperforated panel with partitioned cavity

\section{Conclusions}

In conclusion, present work has been focused on the sound absorption performance of the MPP with partitioned cavity by impedance tube experiments. Comparing with the single-leaf MPP absorber, the MPP with partitioned cavity has two sound absorptions peaks to broaden the bandwidth of the low and middle frequency.

\section{Acknowledgements}

This work was supported by the National Natural Science Foundation of China under Grant No. 11274048, 11102093, Beijing Natural Science Foundation No.8142016 and Project of innovation of Beijing Academy of Science and Technology PXM2014_178304_000004.

\section{References}

[1] K. Sakagami, M. Morimot, M. Yairi. A note on the effect of vibration of a microperforated panel on its sound absorption characteristics [J]. Acoust. Sci. Tech. 2005, 26(2), 204-207.

[2] D. Y. Maa, Theory and design of microperforated panel sound-absorbing constructions, Sci. Sin. 1975, 18, 55-71.

[3] D. Y. Maa, Microperforated-panel wideband absorbers, Noise Cont. Eng [J]. 1987, 29, 77-84.

[4] D. Y. Maa, Potential of microperforated panel absorber[J]. J. Acoust. Soc. Am. 1998, 104, 2861-2864.

[5] K. Sakagami, M. Morimoto, M. Yairi, Double-leaf microperforated panel space absorbers: A revised theory and detailed analysis [J]. Apll. Acoust. Vol.2009, 70, 703-709.

[6] M. Toyoda, R. L. Mu, D. Takahashi, Relationship between Helmholtz-resonance absorptionand panel-type absorption in finite flexible microperforated-panel absorbers[J]. Appl. Acoust.2010, 71, 315-320

[7] H. V. Fuchs, X. Zha, H. D. Drotle. Creating low-noise environments in communication rooms [J]. Appl. Acoust.2001, 62, 1375-1396 .

[8] X. Zha, H. V. Fuchs, H. D. Drotle. Improving the acoustic working conditions for musicians in small spaces [J]. Appl. Acoust. 2002, 63, 203-221 . 
[9] D. Y. Maa, Absorption limit of MPA, ACTA ACUSTICA[J]. 2003, 28(6), 561-562

[10] D.Y. Maa, K. Liu. Sound absorption characteristics of microperforated absorber for random incidence, ACTA ACUSTICA[J]. 2000, 25(4), 289-296.

[11] F. Asdrubali, G. Pispola, Properties of transparent sound-absorbing panels for use in noise barriers [J]. Acoust. Soc. Am, 2007, 121, 214-21

[12] K. Sakagami, M. Morimoto, W. Koike. A numerical study of double-leaf microperforated panel absorbers [J]. Appl. Acoust, 2006, 67, 609-619. 